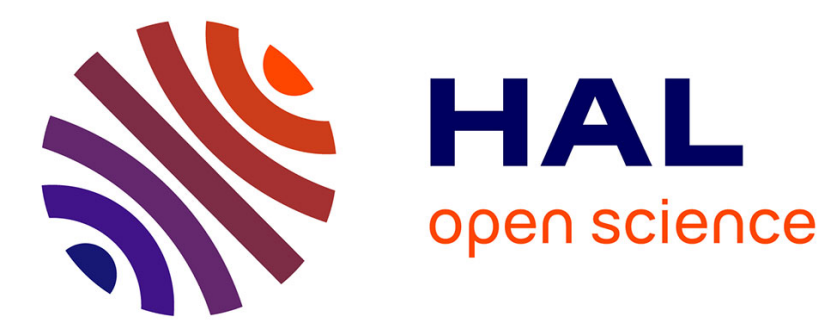

\title{
Enantiopure isothiourea@carbon-based support: stacking interactions for recycling a lewis base in asymmetric catalysis
}

Yu-Chao Yuan, Mariam Abd El Sater, Mohamed Mellah, Nada Jaber, Olivier David, Emmanuelle Schulz

\section{To cite this version:}

Yu-Chao Yuan, Mariam Abd El Sater, Mohamed Mellah, Nada Jaber, Olivier David, et al.. Enantiopure isothiourea@carbon-based support: stacking interactions for recycling a lewis base in asymmetric catalysis. Organic Chemistry Frontiers, In press, 10.1039/d1qo00646k . hal-03282200

\section{HAL Id: hal-03282200 \\ https://hal.science/hal-03282200}

Submitted on 12 Jul 2021

HAL is a multi-disciplinary open access archive for the deposit and dissemination of scientific research documents, whether they are published or not. The documents may come from teaching and research institutions in France or abroad, or from public or private research centers.
L'archive ouverte pluridisciplinaire HAL, est destinée au dépôt et à la diffusion de documents scientifiques de niveau recherche, publiés ou non, émanant des établissements d'enseignement et de recherche français ou étrangers, des laboratoires publics ou privés. 


\section{Enantiopure Isothiourea@Carbon-Based Support: Stacking Interactions for Recycling a Lewis Base in Asymmetric Catalysis}

Received 00th January 20xx, Accepted 00th January 20xx DOI: $10.1039 / x 0 x \times 00000 x$

\author{
Yu-Chao Yuan, ${ }^{a, b, \dagger}$ Mariam Abd El Sater, ${ }^{a, c}$ Mohamed Mellah, ${ }^{a}$ Nada Jaber, ${ }^{c}$ Olivier R. P. David*b \\ and Emmanuelle Schulz*a
}

Dedicated to Dr. Christian Bruneau for his outstanding contribution to catalysis

\section{Introduction}

The use of enantiopure catalysts in synthesis is nowadays obvious for the preparation of valuable scalemic substrates. For cost and environmental motivations, these precious promoters must be readily available and exhibit high activity at low catalytic loading. In this context, their easy recovery for an efficient reuse is also of great value. Numerous procedures have already reported the heterogenization of chiral catalysts involving either covalent or non-covalent binding to many supports. ${ }^{1}$ As highly stable, ideally porous and mechanically resistant supports, carbon materials have been used extensively in catalysis. ${ }^{2}$ They can indeed be modified by covalent bindings ${ }^{3}$ or be involved in $\pi-\pi$ interactions. ${ }^{4}$ In the first case, a covalent link of the chiral catalyst on the support ensures robustness and decreases any risk of leaching, but it can lead to a tedious synthesis for the necessary modification

\footnotetext{
a. Université Paris Saclay, CNRS, Institut de Chimie Moléculaire et des Matériaux d'Orsay, 91405 Orsay, France, emmanuelle.schulz@universite-paris-saclay.fr b. Institut Lavoisier, UMR 8180, Université de Versailles Saint-Quentin-en-Yvelines, Université Paris Saclay, 45 avenue des Etats-Unis, 78035 Versailles, France, olivier.david@uvsq.fr

c. Laboratoire de Chimie Médicinale et des Produits Naturels, Université Libanaise, Faculté des Sciences (I) and PRASE-EDST, Hadath, Beyrouth, Lebanon

+ Present address: Jiangsu Key Laboratory of New Drug Research and Clinical Pharmacy, School of Pharmacy, Xuzhou Medical University, Xuzhou 221004, China. Electronic Supplementary Information (ESI) available: [Experimental procedures, spectroscopic characterization data, copy of NMR and HPLC spectra]. See DOI: $10.1039 / \times 0 \times x 00000 x$
}

of both the ligand and the support. On the contrary, immobilization by non-covalent interactions often means simpler processes and easy recovery of the support, when the catalyst is deactivated. A possible catalyst release must however be taken under consideration. ${ }^{5}$ The chemical modification of carbon supports has already been described for the subsequent covalent grafting of enantiopure organometallic catalysts, ${ }^{6}$ but very few examples have proposed immobilization by non-covalent bonds. ${ }^{7}$ They include the deposit of chiral phosphine-containing catalysts on $\mathrm{CNT}^{7 \mathrm{a}}$ or their assembly onto $\mathrm{rGO}^{7 \mathrm{~d}}$ to realize $\mathrm{Rh}$-promoted asymmetric hydrogenation. Our group described the immobilization of copper bis(oxazoline) complexes on various carbon supports for the enantioselective formation of C-C bonds. ${ }^{7 b}$ More recently, the non-covalent immobilization of $\mathrm{Rh}$ complexes was reported for the asymmetric hydroformylation and implemented in a flow mode. ${ }^{7 c}$ This year, imidazolium salts have also been immobilized by $\pi$-stacking interactions onto carbon materials and these organocatalysts were efficiently reused in the cycloaddition of epoxides with $\mathrm{CO}_{2}{ }^{8}$ Worth mentioning is, in each case, the functionalization of the ligand by a pyrene group as a demonstration of its high affinity with the carbon surface by very stable $\pi$ - $\pi$-interactions. However, and as far as we know, there are no examples dealing with the immobilization of an enantiopure organocatalyst on carbon supports by such reversible interactions. 
Chiral isothioureas (ITUs) ${ }^{9}$ are enantiopure Lewis bases active to promote numerous asymmetric catalytic processes. ${ }^{10,11}$ These catalysts were already immobilized mostly on organic polymeric supports by covalent grafting; they were stable in many efficient batches and even flow processes. ${ }^{12}$ In this context, we describe here the first immobilization of an enantiopure ITU organocatalyst (hyperBTM) on reduced graphene oxide ( $\mathrm{rGO}$ ) as carbon support through $\pi-\pi$ noncovalent interactions. We selected the enantioselective formal [3+2] cycloaddition of ammonium enolates with oxaziridines described by the group of Smith, ${ }^{13}$ to test our procedure. This reaction indeed leads to the synthesis of oxazolidin-4-ones, valuable scaffolds present in natural products such as lipoxazolidinones $\mathrm{A}^{-\mathrm{C}^{14}}$ or simply precursors of chiral $\alpha$ hydroxyacids. While this reaction has never been described under recycling conditions, we report here our efforts to achieve it under heterogeneous conditions on a carbonaceous support, and to evaluate the robustness of the chiral organic catalyst to recovery.

\section{Results and discussion}

We targeted the synthesis of pyr-hyperBTM for its immobilization on $\mathrm{rGO}$ (see Scheme 1). The synthesis of $\mathbf{3}$ was realized following the procedure described by the group of Smith, starting from benzaldehyde $1 .{ }^{15}$ The introduction of the stereogenic centers occurred via the use of $(S)$-proline leading to the formyl-carbamate $\mathbf{2}$ (see Supporting Information for details). The anchoring point for the pyrene ring is chosen far from the active center of the organocatalyst, on the phenyl ring of the benzotetramisole. This strategy has already been illustrated by the immobilization of the enantiomer of compound $\mathbf{3}$ on a Merrifield-type resin, dedicated to the acylative kinetic resolution of alcohols. ${ }^{12 c}$ To this aim, compound $\mathbf{3}$ is functionalized by a propargyl group after demethylation, to give 4 in $42 \%$ yield. On the other side, commercially available pyren-1-yl-methanol is transformed via two consecutive nucleophilic substitutions into the azido derivative 7, possessing a four methylene spacer. A copper(I)promoted azide-alkyne cycloaddition between ITU-alkyne 4 and pyrene-azide 7 afforded the desired functionalized pyrhyperBTM. This pyrene-modified organocatalyst was prepared at the gram scale with an overall yield of $4.1 \%$ from benzaldehyde (see Supporting Information for the detailed complete procedure and characterization).

Immobilization of the pyrene-tagged hyperBTM onto the rGO surface was achieved by impregnation of the carbon suspension with a solution of pyr-hyperBTM in DCM with a mass ratio of $4 / 1 .{ }^{16}$ After sonication for a few minutes and $24 \mathrm{~h}$ of stirring at room temperature, the solid was filtered off, washed with DCM and vacuum-dried. The efficiency of the immobilization was monitored by UV-Vis analyses (see Figure 1 and Supporting Information for details). The comparison with a calibration curve indicated that the entire complex was totally deposited, which was further confirmed by differential weighing. The organocatalyst dispersed as a fine black powder was therefore tested as heterogeneous catalyst.

The catalytic efficiency and the recyclability of pyrhyperBTM@rGO was tested on the asymmetric formal [3+2] cycloaddition of 2-phenylacetic anhydride and rac-3-phenyl-2tosyl-1,2-oxaziridine. ${ }^{13}$ Pyr-hyperBTM was firstly engaged under homogeneous conditions to assess its efficiency against the published values of its unmodified analogue. The reaction is indeed described to deliver the oxazolidin-4-ones anti-10a and syn-10a from racemic 9 and $\mathrm{Cs}_{2} \mathrm{CO}_{3}$ as base, in high yield, with excellent enantiocontrol but a poor diastereoselectivity when hyperBTM is used (Table 1, entry 1 , ref 13 ). We explored the activity of the pyr-hyperBTM precursor 3 under the same conditions, to evaluate the changes induced by the presence of a methoxy substituent on the phenyl ring of the organocatalyst (entry 2).

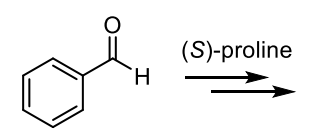

1

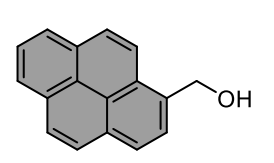

5

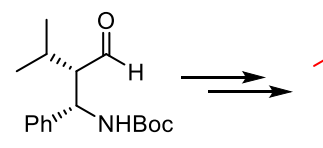

2

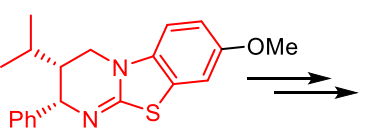

$3.3 \mathrm{~g}$

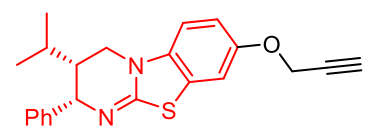

$4 \quad 1.02 \mathrm{~g}$

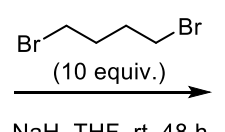

$\mathrm{NaH}, \mathrm{THF}, \mathrm{rt}, 48 \mathrm{~h}$

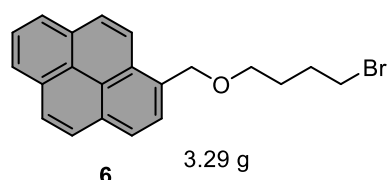

6

$3.29 \mathrm{~g}$

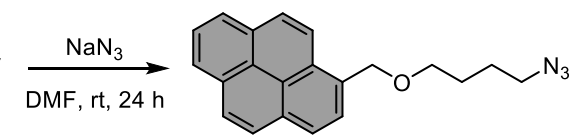

$7 \quad 2.95 \mathrm{~g}$

$88 \%$ yield from 5

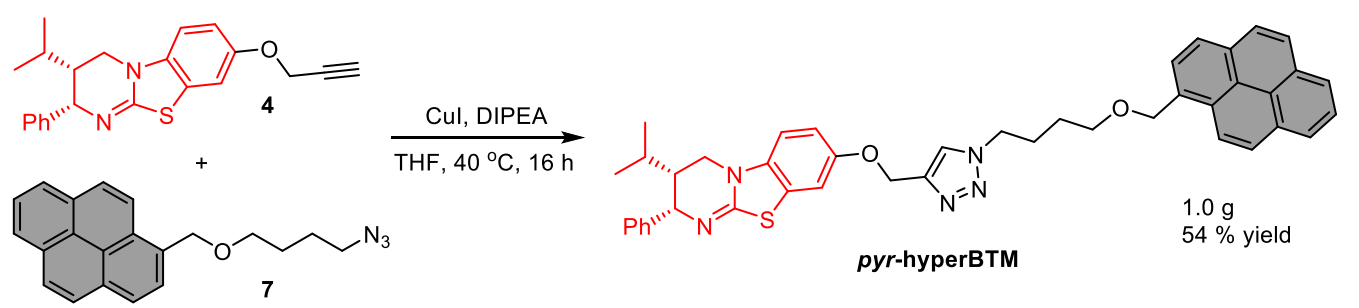


Scheme 1. pyr-hyperBTM synthesis procedure
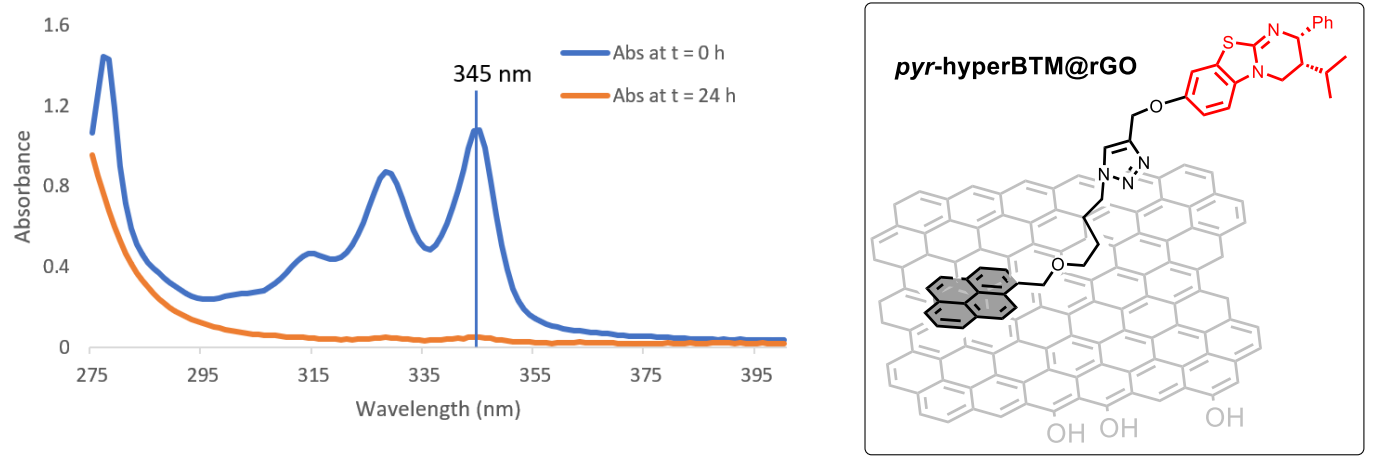

Figure 1.

Immobilization of pyr-hyperBTM on rGO monitored by UV-Vis analyses (blue curve: UV-Vis spectrum for pyr-hyperBTM in DCM. Orange curve: UV-Vis spectrum for pyr-hyperBTM/rGO 1/4 wt/wt in DCM, after $24 \mathrm{~h}$ stirring).

Table 1. Reaction tests in homogeneous and heterogeneous conditions

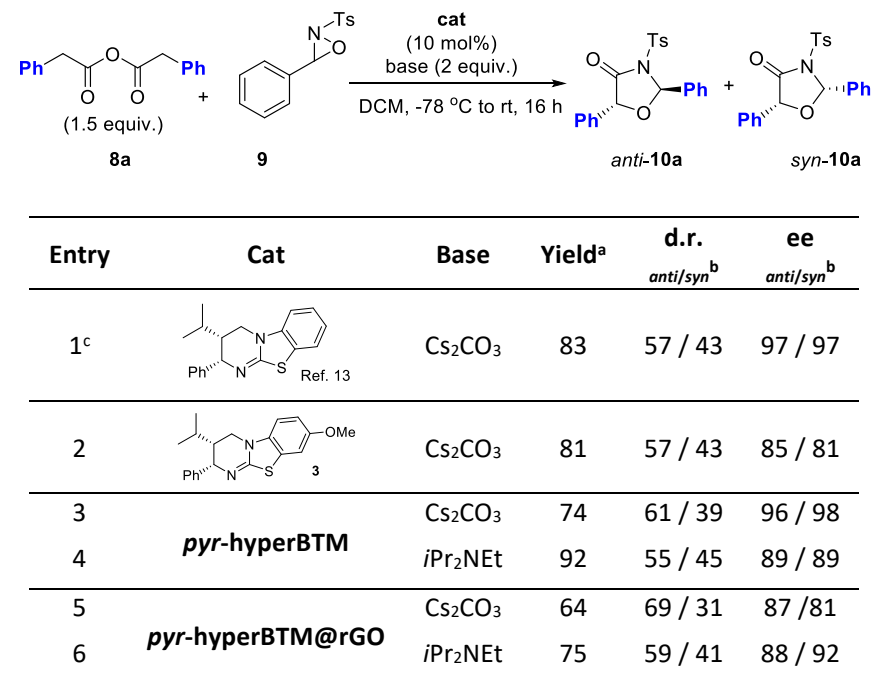

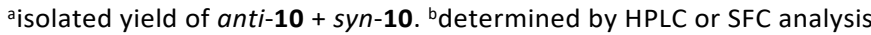
with a chiral stationary phase (see Supporting Information).

This species delivered the target products with similar values in terms of yield and diastereoselectivity but a reduction of the enantioselectivity was observed. Fortunately, high selectivity values were again obtained when pyr-hyperBTM was tested as catalyst in the presence of $\mathrm{Cs}_{2} \mathrm{CO}_{3}$ as base, although both oxazolidin-4-ones were synthesized in only $74 \%$ yield (entry 3 ). The use of an organic base, the Hünig base, was then investigated as soluble species in the medium for limiting mass transfer in the context of the subsequent heterogeneous catalysis. These operating conditions led to an improved activity (92\% yield, entry 4 ) to the detriment of selectivity values. In this last case, both diastereoisomers anti-10a and syn-10a were recovered but contaminated by a slight amount of $\mathrm{N}$-tosylbenzylimine, as by-product presumably arising from oxidation of the organic base.

The reaction was finally performed under heterogeneous conditions, with pyr-hyperBTM@rGO as supported catalyst in the presence of both bases and successful enantioselective catalysis still occurred. The yields were recorded the same reaction time as under homogeneous conditions, revealing a small decrease in activity as is often observed with supported catalysts. The catalyst deposit on rGO slightly improved the diastereoselectivity, but decreased the enantioselectivity value, specifically when $\mathrm{Cs}_{2} \mathrm{CO}_{3}$ was concerned (entries 5-6). The quantity of organocatalyst introduced to carry out this catalytic transformation is not negligible $(10 \mathrm{~mol} \%)$ and we therefore explored the possibility of its recycling to artificially increase the TON. Both the use of the organic or the inorganic base was evaluated for the recovery process and the detailed results are summarized in the Supporting Information (see tables, ESI S14). In all cases, and after the first run, the suspension was left to settle, the supernatant solution was removed by filtration and the remaining solid was washed with DCM, the reaction solvent. A second run was performed after drying the immobilized catalyst under vacuum and addition of new substrates. For instance, $\mathrm{Cs}_{2} \mathrm{CO}_{3}$ possesses the advantage to avoid the formation of the by-product, but as an insoluble species, it is recovered together with the immobilized catalyst. The second run conducted under these conditions and without addition of supplementary base, delivered the desired mixture of diastereoisomers $10 \mathrm{a}$, rid of $\mathrm{N}$-tosylbenzylimine but in only $20 \%$ yield. Addition of $\mathrm{Cs}_{2} \mathrm{CO}_{3}$ before a third use of the catalyst unfortunately did not result in any formation of the targets. A new batch of pyr-hyperBTM@rGO was prepared and a second attempt was completed with the inorganic base, but the workup implied a washing with a mixture of $\mathrm{H}_{2} \mathrm{O}$ /acetone to eliminate all salts. A significant decrease in yield was also observed in this case ( $2^{\text {nd }}$ run, $40 \%$ yield) but the products 
were still isolated in $23 \%$ yield after the third cycle. Nevertheless, the decrease in the enantioselectivity values along with recycling is too important under these conditions and the recycling procedure was thus renewed by using the soluble Hünig base. Both acetone and DCM were tested as solvents to wash the recycled supported catalyst leading to better results. Although a slight decrease in activity could not be avoided, the preservation of the enantioselectivity values was noticeable. Unfortunately, however, the mixture of both diastereoisomers remained contaminated with slight amount of inseparable $\mathrm{N}$-tosylbenzylimine, as by-product.

The procedure implying several washings of the solid catalyst with DCM as reaction solvent and using $i \mathrm{Pr}_{2} \mathrm{NEt}$ as base was thus chosen to carry the reaction with pyr-hyperBTM@rGO in heterogeneous, recycling conditions (Table 2).

Table 2. Recycling tests with pyr-hyperBTM@rGO and $i \mathrm{Pr}_{2} \mathrm{NEt}$ as a base.

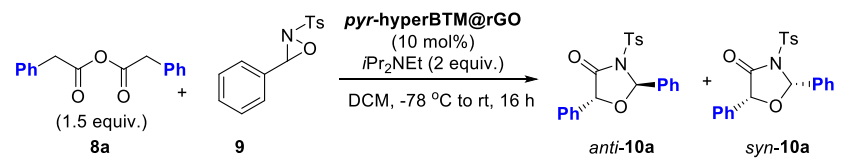

\begin{tabular}{lccc}
\hline & Yield $^{\mathrm{a}}$ & d.r.anti/syn $^{\mathrm{b}}$ & ee $_{\text {anti/syn }}{ }^{\mathrm{b}}$ \\
\hline $1^{\text {st }}$ run & 74 & $57 / 43$ & $92 / 96$ \\
$2^{\text {nd }}$ run & 66 & $64 / 36$ & $90 / 93$ \\
$3^{\text {rd }}$ run & 56 & $62 / 38$ & $87 / 92$ \\
$4^{\text {th }}$ run & 40 & $66 / 34$ & $85 / 87$ \\
$5^{\text {th }}$ run & 29 & $63 / 37$ & $83 / 95$ \\
$6^{\text {th }}$ run & 20 & $68 / 32$ & $74 / 89$ \\
$7^{\text {th }}$ run $^{c}$ & 51 & $67 / 33$ & $91 / 93$
\end{tabular}

aisolated yield of anti-10a + syn-10a. ${ }^{\text {b }}$ determined by HPLC analysis with a chiral stationary phase. ' 2 mg of pyr-hyperBTM added to the spent supported catalyst before performing the $7^{\text {th }}$ run.

The recycling tests were performed at the $0.1 \mathrm{mmol}$ scale, and the supported catalyst pyr-hyperBTM@rGO was engaged seven successive times in reaction. The continuous formation of the target products is observed with each recycling, even if their quantity decreases; after the $2^{\text {nd }}$ run, the imine side product appears as traces together with the expected oxazolidinones. It is important to note that the enantioselectivity values are maintained at a high level, at least until the 5 th use of the catalyst. Finally, and before performing the $7^{\text {th }}$ cycle, a small amount (1/5 of the quantity initially introduced) of fresh pyr-hyperBTM was added on the supported catalyst in reuse. This notably led to a renewed increase in both the yield and also the selectivity of the reaction. Although some performance instability during recycling was observed, possibly due to catalyst degradation over the process by some hydrolysis, ${ }^{17}$ this procedure made it possible to carry out seven runs with notable selectivity, which is unprecedented for this formal $[3+2]$ cycloaddition. A hot filtration test was performed to check if the decrease in yield was not due to a partial leaching of the catalyst from its support. The substrates and the base were added to the supernatant under the usual catalysis conditions and no transformation towards the target product was detected. This test does confirm that the catalysis takes place under heterogeneous conditions.

Finally, pyr-hyperBTM@rGO was used in a similar transformation under these optimized conditions, but different arylacetic anhydrides possessing various structures were successively engaged in the reaction, at each recycling, in a challenging multi-substrate procedure, to widen the scope of the methodology and to further test the stability of the supported catalyst.

Table 3. Homoanhydride substrate scope and recycling tests with pyr-hyperBTM@rGO in a multi-substrate procedure.

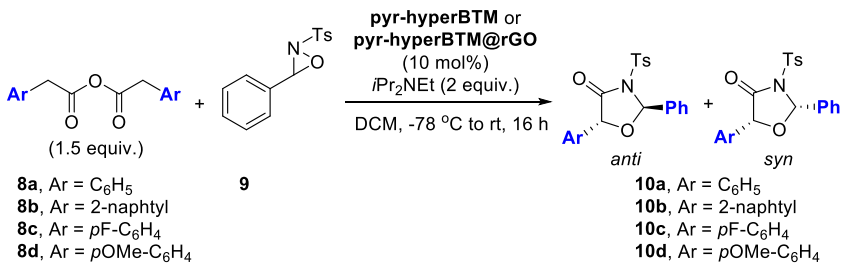

\begin{tabular}{|c|c|c|c|c|c|c|}
\hline Entry & Run & Cat & Substrate & Yielda & $\underset{\text { anti/syn }}{\text { d.r. }}$ & $\underset{\text { anti/syn }}{\text { ee }}$ \\
\hline 1 & - & & $8 a$ & 92 & $55 / 45$ & $89 / 89$ \\
\hline 2 & - & pyr-hyper & $8 b$ & 85 & $58 / 42$ & $79 / 53$ \\
\hline 3 & - & BTM & $8 c$ & 82 & $63 / 37$ & $85 / 60$ \\
\hline 4 & - & & $8 d$ & 90 & $44 / 56$ & $76 / 88$ \\
\hline 5 & $1^{\text {st }}$ & & $8 a$ & 75 & $62 / 38$ & $96 / 91$ \\
\hline 6 & $2^{\text {nd }}$ & pyr-- & $8 b$ & 65 & $80 / 20$ & $86 / 69$ \\
\hline 7 & $3^{\text {rd }}$ & Ypers IVI & $8 c$ & 60 & $44 / 56$ & $72 / 6^{c}$ \\
\hline $8^{d}$ & $4^{\text {th }}$ & & $8 d$ & 30 & $81 / 19$ & $87 / 64$ \\
\hline
\end{tabular}

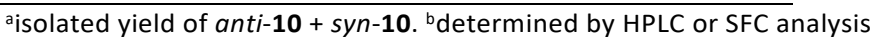

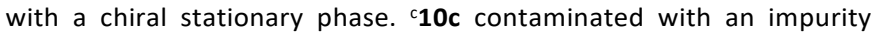
preventing an accurate ee determination of $s y n-10 \mathrm{c} .{ }^{d} \mathrm{Cs}_{2} \mathrm{CO}_{3}$ is used as base.

The three new arylacetic anhydrides were first subjected separately to the homogeneous asymmetric reaction in the presence of pyr-hyperBTM as organocatalyst and yield as selectivity values were determined for comparison (Table 3, entries 2-4). The transformation of these substrates was productive as the target aryl-substituted oxazolidiones were in each case isolated with good to high yields, using Hünig's base. The diastereoselectivity of these reactions are in agreement with published results but the enantioselectivity values turned out to be much weaker than the results reported in the presence of $\mathrm{CS}_{2} \mathrm{CO}_{3} .{ }^{13}$ The ability of pyr-hyperBTM@rGO to catalyze these enantioselective formal cycloadditions by changing the structure of the ammonium enolates at each reuse was finally investigated. After preparation of anti-10a and syn-10a in good isolated yield with high enantioselectivity values, the supported catalyst was washed with DCM and reused for the reaction with 2-(naphthalen-1-yl)acetic anhydride, $\mathbf{8 b}$. Anti-10b and syn-10b were easily recovered in their enantioenriched forms, with $65 \%$ yield, importantly free of any trace of oxazolidinones prepared in the previous cycle. A third recycling of this batch of pyr-hyperBTM@rGo led to the oxazolidinones from the reaction with $\mathbf{8 c}$, however affected by a reduction of the expected values. A final reuse, 
carried out in the presence of $\mathrm{Cs}_{2} \mathrm{CO}_{3}$ as base with $8 \mathbf{d}$ anhydride bearing an electron donor substituent, still made it possible to isolate the targeted products anti-10d and syn-10d with high enantioselectivity values.

\section{Conclusions}

The functionalization of hyperBTM by a pyrene group therefore allowed the proposal of a new method for its recycling, which involves the formation of non-covalent bonds with rGO. These $\pi$-stacking interactions advantageously make it possible to avoid any modification of the support. We have here discussed the fine-tuning of the procedure allowing for the formation of various oxazolidinones from a racemic oxaziridine with arylacetic anhydrides. Two bases were evaluated: the Hünig's base led to high yield and enantioselectivity values in the target products; the use of $\mathrm{Cs}_{2} \mathrm{CO}_{3}$, which is insoluble and accumulates during the recycling of the heterogeneous catalyst made it possible, on the other hand, to avoid the formation of the secondary product, the $\mathrm{N}$-tosylbenzylimine. The catalytic reaction is nevertheless carried out under operating conditions matching those used in the homogeneous phase while maintaining high activity and selectivity. The recovery of the catalyst before its re-engagement in a new reaction is simply carried out by its filtration and further washing with the reaction solvent. Its effectiveness has been demonstrated by its reuse in 7 successive cycles of catalysis and also in a multi-substrate procedure (4 cycles) during which the structure of the anhydride reagent was changed at each recycling. Although a decrease in the activity and selectivity values of the spent catalyst could not be avoided, this is the first demonstration of its reuse in this formal cycloaddition reaction. To our knowledge, this study is also the first use of a chiral organic catalyst under these conditions, among the very few reports which deal with non-covalent immobilization by $\pi$-stacking of asymmetric organometallic catalysts. This study paves the way for the application of this procedure to promote other catalytic reactivities by these chiral isothiourea species, immobilized alone, or with other catalysts, towards asymmetric multicatalysis.

\section{Conflicts of interest}

There are no conflicts to declare.

\section{Acknowledgements}

We acknowledge Université Paris Saclay, CNRS, Ministère de l'Europe et des Affaires Etrangères (bourse d'excellence "Eiffel") and Charm3At LabEx (ANR-11-LABEX-0039) for financial support.

\section{Notes and references}

1 a) C. Baleizão and H. Garcia, Chiral Salen Complexes: An Overview to Recoverable and Reusable Homogeneous and Heterogeneous Catalysts, Chem. Rev., 2006, 106, 3987; b) J. M. Fraile, J. I. García and J. A. Mayoral, Recent advances in the immobilization of chiral catalysts containing bis(oxazolines) and related ligands, Coord. Chem. Rev., 2008, 252, 624; c) A. Zulauf, M. Mellah, X. Hong and E. Schulz, Recoverable chiral salen complexes for asymmetric catalysis: recent progress, Dalton Trans., 2010, 39, 6911.

2 a) V. Georgakilas, M. Otyepka, A. B. Bourlinos, V. Chandra, N. Kim, K. C. Kemp, P. Hobza, R. Zboril and K. S. Kim, Functionalization of Graphene: Covalent and Non-Covalent Approaches, Derivatives and Applications, Chem. Rev., 2012, 112, 6156; b) A. Schaetz, M. Zeltner and W. J. Stark, Carbon Modifications and Surfaces for Catalytic Organic Transformations, ACS Catal., 2012, 2, 1267; c) M. R. Axet, O. Dechy-Cabaret, J. Durand, M. Gouygou and P. Serp, Coordination chemistry on carbon surfaces, Coord. Chem. Rev., 2016, 308, 236.

3 a) A. J. Clancy, H. Au, N. Rubio, G. O. Coulter and M. S. P. Shaffer, Understanding and controlling the covalent functionalisation of graphene, Dalton Trans., 2020, 49, 10308; b) A. Khazaee, R. Jahanshahi, S. Sobhani, J. Skibsted and J. M. Sansano, Immobilized piperazine on the surface of graphene oxide as a heterogeneous bifunctional acid-base catalyst for the multicomponent synthesis of 2-amino-3cyano-4H-chromenes, Green Chem., 2020, 22, 4604.

4 a) G. Liu, B. Wu, J. Zhang, X. Wang, M. Shao and J. Wang, Controlled Reversible Immobilization of $\mathrm{Ru}$ Carbene on Single-Walled Carbon Nanotubes: A New Strategy for Green Catalytic Systems Based on a Solvent Effect on $\pi-\pi$ Interaction, Inorg. Chem., 2009, 48, 2383; b) C. Vriamont, M. Devillers, O. Riant and S. Hermans, Catalysis with Gold Complexes Immobilised on Carbon Nanotubes by $\pi-\pi$ Stacking Interactions: Heterogeneous Catalysis versus the Boomerang Effect, Chem. Eur. J., 2013, 19, 12009; c) M. Keller, V. Colliere, O. Reiser, A.-M. Caminade, J.-P. Majoral and A. Ouali, Pyrene-Tagged Dendritic Catalysts Noncovalently Grafted onto Magnetic Co/C Nanoparticles: An Efficient and Recyclable System for Drug Synthesis, Angew. Chem. Int. Ed., 2013, 52, 3626; d) S. Sabater, J. A. Mata and E. Peris, Catalyst Enhancement and Recyclability by Immobilization of Metal Complexes onto Graphene Surface by Noncovalent Interactions, ACS Catal., 2014, 4, 2038; e) L. Zhang, W. Zhang, P. Serp, W.-H. Sun and J. Durand, Ethylene Polymerization Catalyzed by Pyrene-Tagged Iron Complexes: The Positive Effect of $\pi$-Conjugation and Immobilization on Multiwalled Carbon Nanotube, ChemCatChem, 2014, 6, 1310 ; f) S. Sabater, J. A. Mata and E. Peris, Immobilization of Pyrene-Tagged Palladium and Ruthenium Complexes onto Reduced Graphene Oxide: An Efficient and Highly Recyclable Catalyst for Hydrodefluorination, Organometallics, 2015, 34, 1186; g) H. Nasrallah, S. Germain, P. Queval, C. Bouvier, M. Mauduit, C. Crévisy and E. Schulz, Non covalent immobilization of pyrene-tagged ruthenium complexes onto graphene surfaces for recycling in olefin metathesis reactions, J. Mol. Cat. A: Chemical, 2016, 425, 136; h) S. RuizBotella and E. Peris, Immobilization of Pyrene-Adorned $\mathrm{N}$-Heterocyclic Carbene Complexes of Rhodium(I) on Reduced Graphene Oxide and Study of their Catalytic Activity, ChemCatChem, 2018, 10, 1874; i) P. Ballestin, D. Ventura-Espinosa, S. Martín, A. Caballero, J. A. Mata and P. J. Pérez, Improving Catalyst Activity in Hydrocarbon Functionalization by Remote Pyrene-Graphene Stacking, Chem. Eur. J., 2019, 25, 9534.

5 a) J. M. Fraile, J. I. García and J. A. Mayoral, Noncovalent Immobilization of Enantioselective Catalysts, Chem. Rev., 2009, 109, 360; b) V. I. Parvulescu and H. García, Heterogeneous catalysis based on supramolecular association, Catal. Sci. Technol., 2018, 8, 4834.

6 a) A. Zammataro, C. M. A. Gangemi, A. Pappalardo, R. M. Toscano, R. Puglisi, G. Nicotra, M. E. Fragalà, N. Tuccitto and 
G. T. Sfrazzetto, Covalently functionalized carbon nanoparticles with a chiral Mn-Salen: a new nanocatalyst for enantioselective epoxidation of alkenes, Chem. Commun., 2019, 55, 5255; b) M. Azlouk, M. Durmaz, E. Zor and H. Bingol, Graphene-based recyclable and bifunctional heterogeneous chiral catalyst for direct asymmetric aldol reaction, Mater. Chem. Phys., 2020, 239, 122298.

7 a) L. Xing, J.-H. Xie, Y.-S. Chen, L.-X. Wang and Q.-L. Zhou, Simply Modified Chiral Diphosphine: Catalyst Recycling via Non-covalent Absorption on Carbon Nanotubes, Adv. Synth. Catal., 2008, 350, 1013; b) D. Didier and E. Schulz, $\pi$-Stacking interactions at the service of [Cu]-bis(oxazoline) recycling, Tetrahedron: Asymm., 2013, 24, 769; c) A. Cunillera, C. Blanco, A. Gual, J. M. Marinkovic, E. J. Garcia-Suarez, A Riisager, C. Claver, A. Ruiz and C. Godard, Highly Efficient Rh-catalysts Immobilised by $\pi-\pi$ Stacking for the Asymmetric Hydroformylation of Norbornene under Continuous Flow Conditions, ChemCatChem, 2019, 11, 2195. d) E.-J. Hao, G.-X. Li, Z.-Z. Lv, F.-S. Li, Y.-Q. Chen, S.-J. Lin, C.-Z. Shib and L. Shi, "In situ immobilization" of a multicomponent chiral catalyst (MCC) via non-covalent interactions for heterogeneous asymmetric hydrogenation reactions, Org. Chem. Front., 2020, 7, 345

8 M. Y. Souleymanou, F. El-Ouahabi, A. M. Masdeu-Bultó and C. Godard. Cooperative NHC-based Catalytic System Immobilised onto Carbon Materials for the Cycloaddition of CO2 to Epoxides, ChemCatChem, 2021, 13, 1706.

9 V. B. Birman and X. Li, Benzotetramisole: A Remarkably Enantioselective Acyl Transfer Catalyst, Org. Lett., 2006, 8, 1351.

10 a) J. Merad, J.-M. Pons, O. Chuzel and C. Bressy, Enantioselective Catalysis by Chiral Isothioureas, Eur. J. Org. Chem., 2016, 5589; b) V. B. Birman, Amidine-Based Catalysts (ABCs): Design, Development, and Applications, Aldrichimica acta, 2016, 49, 23; c) A. Biswas, H. Mondal and M. S. Maji, Synthesis of heterocycles by isothiourea organocatalysis, Heterocycles, 2020, 57, 3818; d) C. McLaughlin and A. D. Smith, Generation and Reactivity of C(1)-Ammonium Enolates by Using Isothiourea Catalysis, Chem. Eur. J., 2021, 27, 1533; e) J. Bitai, M. T. Westwood and A. D. Smith, $\alpha, \beta-$ Unsaturated acyl ammonium species as reactive intermediates in organocatalysis: an update, Org. Biomol. Chem., 2021, 19, 2366.

11 a) H. Liu, A. M. Z. Slawin and A. D. Smith, IsothioureaCatalyzed Enantioselective Synthesis of Tetrahydro- $\alpha-$ carbolinones, Org. Lett., 2020, 22, 1301; b) E. S. Munday, M. A. Grove, T. Feoktistova, A. C. Brueckner, D. M. Wallen, C. M. Young, A. M. Z. Slawin, A. D. Campbell, P. H.-Y. Cheong and A. D. Smith, Isothiourea-Catalyzed Atropselective Acylation of Biaryl Phenols via Sequential Desymmetrization/Kinetic Resolution, Angew. Chem. Int. Ed., 2020, 59, 7897; and references cited therein.

12 a) J. Izquierdo and M. A. Pericas, A Recyclable, Immobilized Analogue of Benzotetramisole for Catalytic Enantioselective Domino Michael Addition/Cyclization Reactions in Batch and Flow, ACS Catal., 2016, 6, 348; b) S. Wang, J. Izquierdo, C. Rodríguez-Escrich and M. A. Pericàs, Asymmetric $[4+2]$ Annulation Reactions Catalyzed by a Robust, Immobilized Isothiourea, ACS Catal., 2017, 7, 2780; c) R. M. Neyyappadath, R. Chisholm, M. D. Greenhalgh, C. RodríguezEscrich, M. A. Pericàs, G. Hähner and A. D. Smith, Acylative Kinetic Resolution of Alcohols Using a Recyclable PolymerSupported Isothiourea Catalyst in Batch and Flow, ACS Catal., 2018, 8, 1067; d) N. R. Guha, R. M. Neyyappadath, M. D. Greenhalgh, R. Chisholm, S. M. Smith, M. L. McEvoy, C. M. Young, C. Rodríguez-Escrich, M. A. Pericàs, G. Hähner and A. D. Smith, Evaluating polymer-supported isothiourea catalysis in industrially-preferable solvents for the acylative kinetic resolution of secondary and tertiary heterocyclic alcohols in batch and flow, Green Chem., 2018, 20, 4537; e) J. Lai, R. M. Neyyappadath, A. D. Smith and M. A. Pericàs, Continuous Flow Preparation of Enantiomerically Pure BINOL(s) by Acylative Kinetic Resolution, Adv. Synth. Catal., 2020, 362, 1370 ; f) S. Qu, S. M. Smith, V. Laina-Martín, R. M. Neyyappadath, M. D. Greenhalgh and A. D. Smith, Isothiourea-Catalyzed Acylative Kinetic Resolution of Tertiary $\alpha$-Hydroxy Esters, Angew. Chem. Int. Ed., 2020, 59, 16572; g) A. Brandolese, M. D. Greenhalgh, T. Desrues, X. Liu, S. Qu, C. Bressy and A. D. Smith, Horeau amplification in the sequential acylative kinetic resolution of $( \pm)$-1,2-diols and ( \pm )1,3-diols in flow, Org. Biomol. Chem., 2021, 19, 3620.

13 S. R. Smith, C. Fallan, J. E. Taylor, R. McLennan, D. S. B. Daniels, L. C. Morrill, A. M. Z. Slawin and A. D. Smith, Asymmetric Isothiourea-Catalysed Formal [3+2] Cycloadditions of Ammonium Enolates with Oxaziridines, Chem. Eur. J., 2015, 21, 10530.

14 V. R. Macherla, J. Liu, M. Sunga, D. J. White, J. Grodberg, S. Teisan, K. S. Lam and B. C. M. Potts, Lipoxazolidinones A, B, and C: Antibacterial 4-Oxazolidinones from a Marine Actinomycete Isolated from a Guam Marine Sediment, J. Nat. Prod., 2007, 70, 1454.

15 L. C. Morrill, J. Douglas, T. Lebl, A. M. Z. Slawin, D. J. Fox and A. D. Smith, Isothiourea-mediated asymmetric Michaellactonisation of trifluoromethylenones: a synthetic and mechanistic study, Chem. Sci., 2013, 4, 4146.

16 M. Abd El Sater, M. Mellah, D. Dragoe, E. Kolodziej, N. Jaber and E. Schulz, Chiral Chromium Salen@rGO as Multipurpose and Recyclable Heterogeneous Catalyst, Chem. Eur. J., 2021, doi.org/10.1002/chem.202101003.

17 a) For a potential deactivation pathway by hydrolysis, see $X$. Li, H. Jiang, E. W. Uffman, L. Guo, Y. Zhang, X. Yang and V. B. Birman, Kinetic Resolution of Secondary Alcohols Using Amidine-Based Catalysts, J. Org. Chem., 2012, 77, 1722; b) For a possible oxidation of the sulfur atom of the ITU catalyst, see A. -N. El-Shorbagi, S. -I. Sakai, M. A. El-Gendy, N. Omar and H. H. Farag, Imidazo\{2, 1-b]benzothiazoles. II. Synthesis and Anti-inflammatory Activity of Some Imidazo[2, 1-b]benzothiazoles, Chem. Pharm. Bull., 1989, 37, 2971. 